\title{
AN ASSISTIVE COBOT FOR AID IN SELF CARE ACTIVITIES
}

\author{
Laurent Jabre, Rebecca McGrew, R. Brent Gillespie*, Patrick Goleski
}

\author{
Department of Mechanical Engineering \\ University of Michigan \\ 1231 Beal Avenue \\ Ann Arbor, MI 48109 \\ (734) 647-6907 (phone), (734) 764-4256 (fax) \\ enzo, rmcgrew, brentg, pgoleski@umich.edu \\ * corresponding author
}

\begin{abstract}
This paper reports on the design and development of a robotic device intended to assist persons with motor impairments. In the new design, assist is not offered in the production of motion of the user's limb, per the traditional approach using a robot configured as a powered orthosis. Rather, assist is offered in the determination of allowed motion, using the recently developed technology of cobots. A cobot is presented that is capable of guiding (but not producing) motions of the hand in a spherical workspace centered about an elbow resting on a table. Arbitrarily shaped paths embedded with the spherical workspace that feel to the user like guiding channels, say from soup bowl to mouth, may be programmed and user-selected. The cobot is able to produce forces perpendicular to the programmed path to suppress tremor or unintended involuntary movement while, by design, the cobot is unable to produce forces that oppose user motion in the intended directions (along the programmed path). The design of an assistive cobot is presented, and certain aspects of the mechanical and control design are discussed in detail. A numerical analysis and experimental characterization of rolling contact, which is elemental to cobot function, is outlined. Various advantages are expected to accrue from the application of cobots as assistive technology, given that the user remains in command of the basic production of motion.
\end{abstract}

Classification Codes: C2, H1

Keywords: Human-machine interface, Mechatronic assistants, Service robots, Haptic interface

\section{INTRODUCTION}

COBOTS (COllaborative roBOTS) are a new family of robotic devices designed to share a manipulation task with a human user. Rather than automating the task and supplanting the human like a traditional robot, a cobot grasps a workpiece together with a human user and shares in the determination of its motion. The cobot cooperates with the user, contributing assistance in the form of forces that resist unwanted motion. These programmable forces are coordinated so that a useful mental map of virtual objects of various shapes is created within the mind of the user. The user recognizes these objects by feel and uses their positions and shapes to constrain workpiece orientation or motion during the manipulation, which simplifies execution and facilitates task completion. Because of their utility in aiding manipulation, these object images are called "virtual fixtures". For example, a cobot can make the workpiece behave as if it were moving along a straight-edge. Alternatively, the cobot can allow free motion within a certain specified region, bordered with virtual walls. Notably, all virtual fixture geometries are defined in software and may be re-configured at the touch of a button.

Elemental to cobot technology is the use of rolling wheels under computer-controlled steering to realize the forces of constraint underlying a virtual fixture. This use of steered wheels renders cobots inherently passive - they are not able to move on their own. It is up to the user to produce all motion of the workpiece. This provides a very important safety guarantee that is not available from traditional robotic or haptic interface devices. Colgate and Peshkin (1996), (Peshkin et.al. 2001) first introduced cobots in 1995 as a new technology for the materials handling industry, especially for parts handling in automobile assembly. One of the most natural applications of cobot technology, however, has not been addressed to date: for assisting persons with motor impairments.

The intrinsic properties of a cobot, namely its safety and ability to create virtual fixtures of arbitrary shape, make cobot technology an ideal candidate for use as an assistive device for persons with motor control deficiencies. Use of a cobot is intuitive because of its 
reliance on common experience in the physical world: manipulation of objects and fixtures that do not interpenetrate. For a person whose motor behavior is impeded by a severe tremor or any component of uncoordinated movement, a cobot can disallow the undesired motions while allowing the desired motions for a given task. Also, the rehabilitating stroke patient might find virtual fixtures a natural form of therapy. One by one, the fixtures could be removed as rehabilitation progresses. Cobots also satisfy another major concern in assistive technology: whether the user perceives the technology as truly subservient. Robotic technology to date has not been able to provide a human interface that suitably renders the robot "always in service" while maintaining tight control. Robots, by design, are generally autonomous in nature whereas cobots are designed as assist technology from their conception.

\subsection{Cobot Technology}

Cobots are based on Continuously Variable Transmissions (CVTs). A CVT is an apparatus that couples a pair of speeds, constraining them mechanically to a particular ratio that is under computer control. Translational CVTs couple translational speeds whereas rotational CVTs couple rotational speeds. Generally, infinitely variable transmissions are required to realize cobots, or CVTs with transmission ratios that can be varied from large negative numbers through zero to large positive numbers (in practice, infinite ratio values are not necessary). Closed loop control ensures that certain non-ideal behavior does not contribute to a loss of performance.

A simple steered wheel can be considered a translational CVT. It constrains velocity in one direction to be proportional to velocity in the orthogonal direction by a factor determined by the instantaneous steering angle. The steering orients the rolling direction in the frame determined by the two basis directions. Figure 1 shows a single steered wheel whose rolling and axial directions orient a frame called $(\perp, \|)$ in a framed called $(1,2)$. The components of a traction force transmitted across the rolling contact may be resolved into the components $\mathbf{f}_{\perp}$ and $\mathbf{f}_{\|}$as shown. The force $\mathbf{f}_{\|}$is always tangent to the path taken by the rolling contact in the ideal case.

For a complete discussion of the translational and rotational CVTs used in cobots, including an analysis of the contact mechanics governing non-ideal behavior in the rotational CVT, see (Gillespie, 2002) and for an introduction to cobot technology, see (Peshkin et.al. 2001). For the cobot with spherical workspace presented in this paper, only a single translational CVT, or single steered rolling wheel is needed.

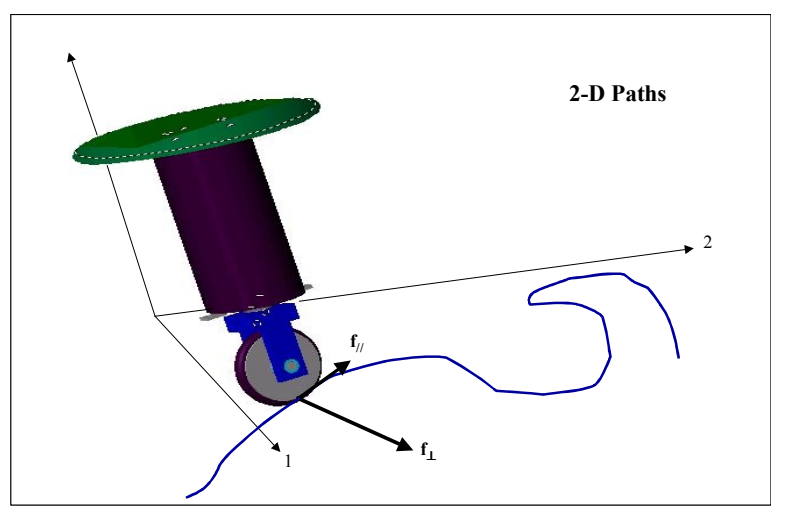

Figure 1 A single steered wheel interpreted as a translational CVT

CVTs (whether translational or rotational) are used together with a network of joints and links to constrain the motion of the cobot end-effector. By virtue of the nonholonomic (motion rather than configuration) nature of the constraints contributed by each of the CVTs, the allowed direction of motion of the endeffector is constrained, not the configuration. Also, since each CVT steering angle (transmission ratio) is motorized, the allowed direction of motion of the endeffector is determined instantaneously through computer control.

Cobots have two basic modes of operation, "virtual caster mode" and "path mode", which are invoked variously by a controller, according logical functions of sensed position. Virtual caster mode requires use of a force transducer located at or near the cobot's grasp point, whereas virtual path mode does not. Switching between the two modes is used to realize virtual walls, which are the basic components of any virtual fixture.

Our objective is to create a simple device based on cobot principles that will assist motor impaired people to feed themselves. The idea is to create a mechanism that will guide the user's hand from his plate to his mouth and back. This apparatus will be inherently safe by guiding the hand trajectory while leaving motion control to the user; i.e.: the motion is produced only as a result of the user's volition.

\section{SIGNIFICANCE}

Over two million Americans have to cope with some form of involuntary muscle movement such as tremor or jerking (Stolov, 1981). Persons who have Parkinson's disease, Huntington's disease, Multiple 
Sclerosis, and Cerebral Palsy make up a large percentage of individuals who have some form of involuntary muscle movement. Although treatments such as drugs or deep brain stimulation may be able to alleviate certain symptoms, at present there is no medical cure for these conditions (Larsen, 1997).

Involuntary muscle control often has a profound effect on a person both emotionally and functionally. Feelings of embarrassment and social isolation may develop, leading to an altered lifestyle. Depression, irritability, and apathy can develop after the onset of involuntary muscle movement (Larsen, 1997). A person's response to involuntary muscle control can have a dramatic effect on the way the disorder affects their life. Re-establishing a person's functionality in daily tasks will allow the individual to gain their pride and independence back. When a person's movements are controlled to achieve the desired outcome, the individual will feel a sense of accomplishment.

We intend to explore the degree to which a cobot can help an individual dealing with involuntary muscle movement regain a sense of accomplishment. Rather than automating the task like a traditional robot, cobots are designed to share a manipulation task with a human user. The cobot helps to guide the human muscle to perform a desired task. The human provides the necessary power, while the cobot directs this power into the desired motion. Since a cobot cannot move on its own, a level of safety is provided that is not available from a traditional robotic device.

Figure 2 contrasts a cobot to a traditional robot. A force from a human user activates the cobot to perform a particular task. The cobot resists any forces perpendicular to a predetermined direction while allowing forces parallel to the predetermined direction to produce motion. Since the input to the cobot is human force, the cobot will not move without human interaction. In contrast, a switch, controlled by a human user, activates a robot to perform a desired task. The robot performs the programmed activity based on the switch signal. Unlike the cobot, a robot does not require much interaction from the human user.

\section{DESIGN}

Assuming that the wrist joint remains fixed, the hand moves in a taskspace that is a semi-sphere centered at the elbow on the table-top. A mechanical schematic is presented in Figure 3 showing a long slender rod connected to ground through a gimble-joint. The endeffector which carries a workpiece (perhaps a spoon) is the end of the long portion of the rod.

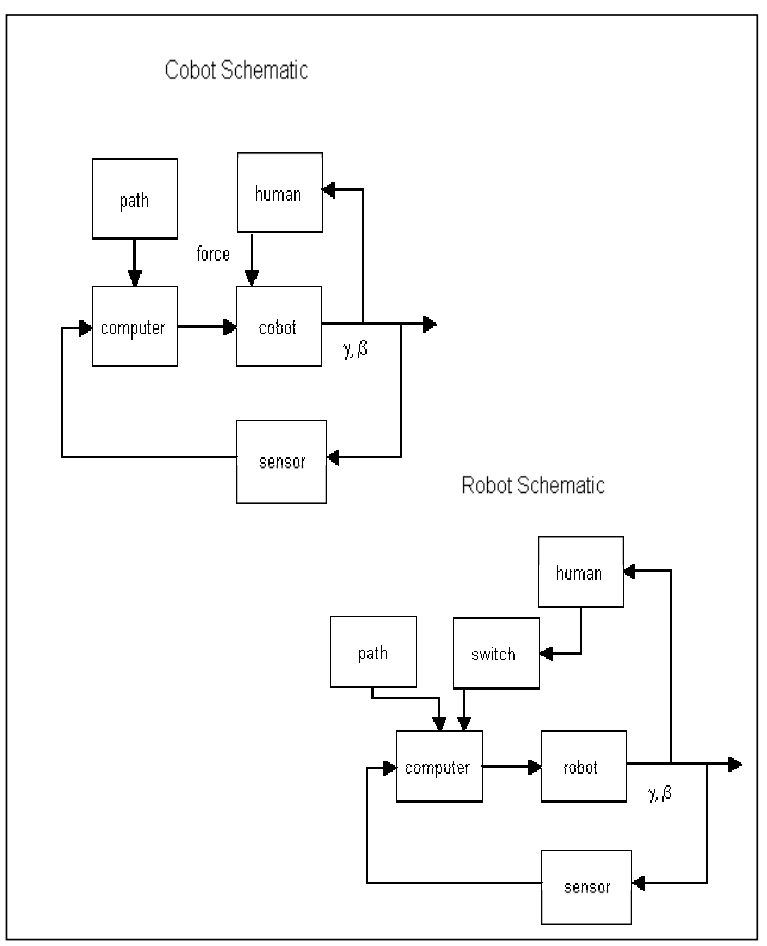

Figure 2: Block Diagrams comparing robots to cobots

This is where the cobot and human user both grasp the workpiece. (Alternatively, the user may grasp the cobot by a handle here and the cobot may hold the workpiece.) The user's elbow rests on a pad configured at or near the gimble center. The shorter portion of the rod extends from the opposite side of the gimble center, effectively below a table-top. This portion becomes an extension of the arm to the opposite side of the elbow. To the end of this portion, a wheel is attached with its steering axis co-linear to the rod and with that steering axis motorized. Consequently, "guidance" of the wheel will directly dictate a symmetric and proportional "guidance" to the hand. Effectively, the taskspace of the wheel is on a smaller semi-sphere than that of the hand. A solid semi spherical surface (not shown in Figure 3) provides a rolling surface for the wheel. Finally, a force sensor between the hand grasp-point and the gimble-joint can be used to sense user intended motion direction for the purpose of realizing caster-control modes.

Once the steered wheel is placed in contact with a rolling surface and under computer control, this mechanism is a cobot and can be thought of as a translational CVT with a spherical taskspace. When steering the wheel through a given trajectory we are imposing a specific path on the user's hand. 


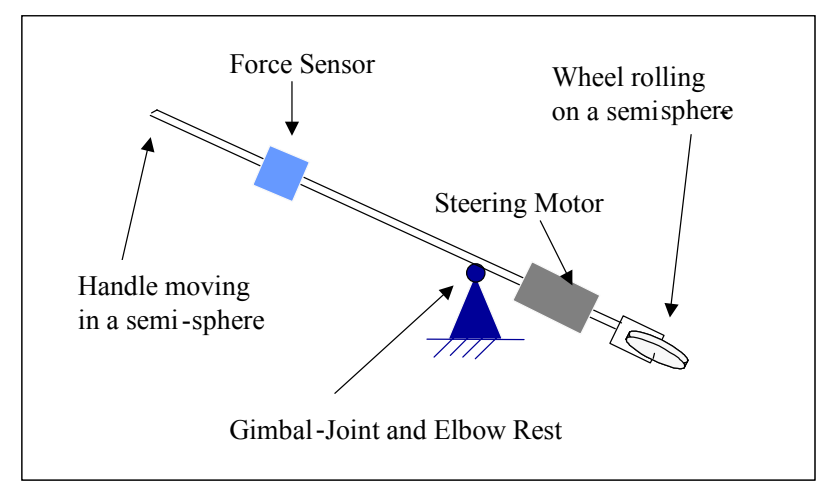
workspace

Figure 3 Schematic of a cobot with spherical

Figure 4 presents the mechanical design drawings of the cobot, with the important components labeled. The interface with the user is the slender rod or lever arm extending above the gimble. The lever arm transmits forces from the user to a wheel that rolls on a semi-spherical surface. The rolling direction of the wheel defines tangent directions along a path with programmable shape inside the semi-spherical rolling surface. The lever arm is also designed to mount a device, such as a brush or eating utensil, for use in performing a specific activity. The lever arm is connected to a gimble that allows movement of the arm in two rotations. Angular encoders in the gimble track the orientation of the lever arm throughout the activity. A microprocessor compares the position of the wheel of the device to a point on the desired path and uses a feedback control law to steer the wheel contact point back onto the desired path if it has strayed away. For a complete discussion of the control methodology, see (Gillespie et.al., 2001). The redirection of the force is obtained through the use of a motor that steers the wheel in the desired direction.

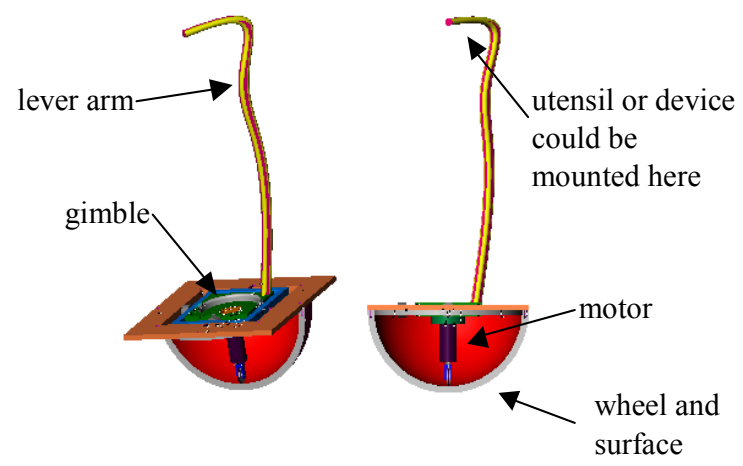

Figure 4: Main Components of Cobot
Figure 5 presents further detail on the mechanical design as well as a photograph of a semi-functional mock up, which we call the "Elbow Cobot".

The gimbal-joint features two orthogonal axes connecting the lever-arm, steering motor and wheel assembly first to an intermediate frame and then to the base frame and semi-sphere assembly. Two encoders read the rotation angles of the gimble joint and thus give the position of the wheel on the semi sphere. The gimbal-joint as well as the wheel incorporate preloaded axial bearings to support radial and axial forces, but allow free rotation. The rotation of the wheel about its (steered) axis is not motorized nor braked. Steering of the wheel is achieved by direct drive using an axially mounted motor and is measured by a third encoder. Using this encoder, the steering angle is placed under PID position control. Fine threads on the gimble bearing mounts allow centering of the gimbal and large diameter threads on an assembly that mounts the motor onto the innermost gimble frame allow adjustable compression of the wheel on the solid semi-sphere and thereby determine the normal contact force.

At present, no force transducer is used in the design and only path mode is available. However, the virtual path or channel is all that we are seeking to apply as assistance or tremor supression. Our first goal is to create one-dimensional paths on the hemisphere that rely only on this path mode.

\section{ROLLING CONTACT MECHANICS}

A very important consideration in the design of the elbow cobot is the choice of material and geometry in the rolling contact between steered wheel and semisphere. The rolling mechanics determine the motor and motor drive transmission requirements and determine the maximum force $\mathbf{f} \perp$ that may be applied against the edges of a path or virtual wall beyond which gross slip will occur.

Additionally, the rolling contact mechanics determine the minimum force $\mathbf{f}_{\|}$that must be applied to induce motion tangent to a path or virtual wall (or in any direction during caster mode). Figure 6 shows a top view of the contact patch between the steered wheel and semi-spherical rolling surface. Also indicated in

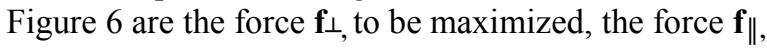
to be minimized, and the steering torque $\tau_{\mathrm{s}}$, also to be minimized. In recent years, most cobots have been designed and built using roller-blade wheels, which produce elliptical contact patch shapes in which the long ellipse axis is aligned with the rolling direction of the wheel. 

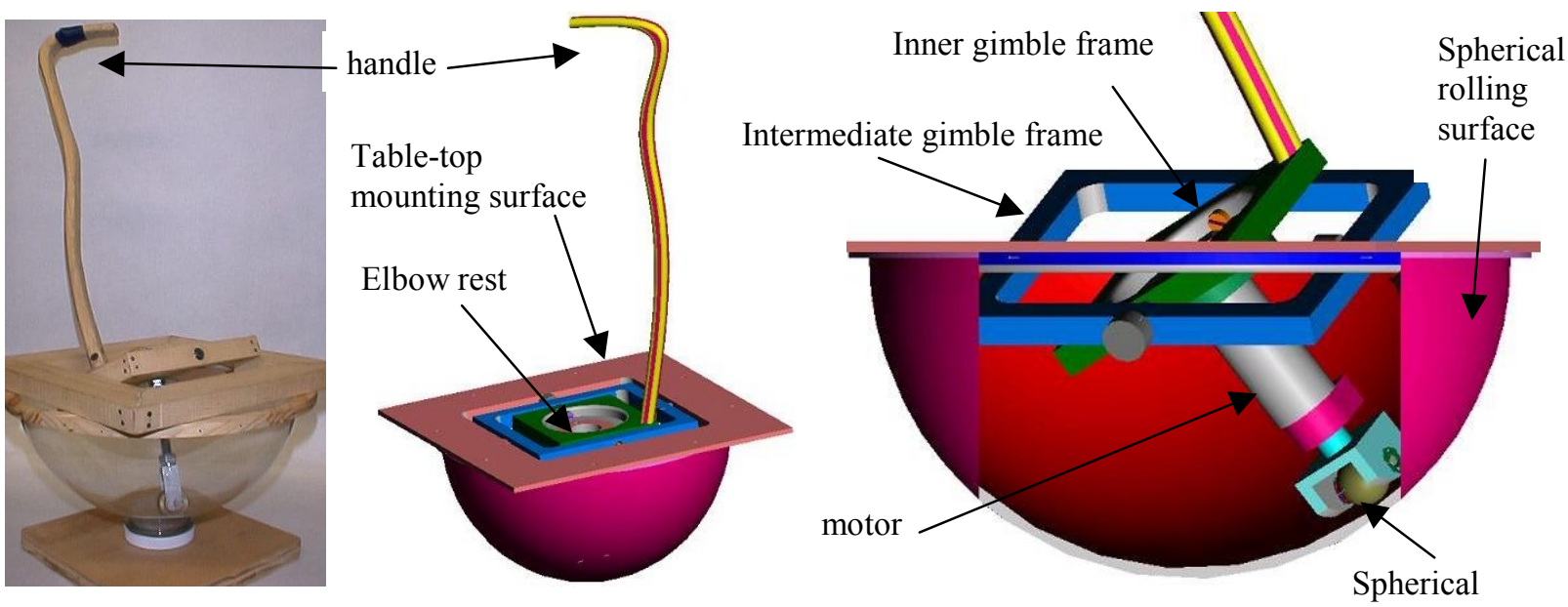

Figure 5 Wooden semi-functional mock-up and design drawing details wheel

Such a shape might maximize $\mathbf{f}_{\perp}$ and minimize $\mathbf{f}_{\|}$, but it would also increase $\tau_{\mathrm{s}}$, requiring a larger steering motor, compared to a circular contact patch. A pair of wheel and rolling surface materials with a high coefficient of friction would be desirable to support lateral loads $(\mathbf{f} \perp)$ but stiff surfaces and small contact patch sizes would be desirable to minimize the rolling resistance $\left(\mathbf{f}_{\|}\right)$and steering torque $\left(\boldsymbol{\tau}_{\mathbf{s}}\right)$. It is apparent that a rather complicated set of design tradeoffs exist.

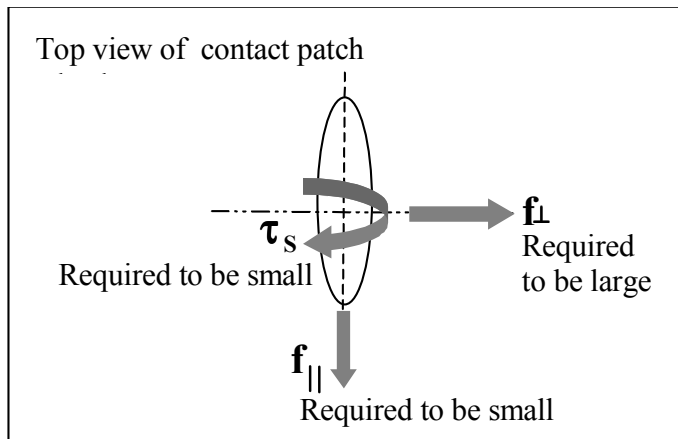

Figure 6: Three friction forces and their requirements

Given that the contact mechanics play such an important role in the determination of the performance of any cobot design, we have undertaken both a numerical analysis and an experimental characterization of rolling contact. With these two endeavors, we hope to optimally settle the various design tradeoffs and produce a set of cobot design directives. Generally, an analytical solution is not available and either numerical analysis or experimental characterization is needed, since the conditions of rolling or slip at the various points within a contact patch depend on both kinematics and kinetics and in turn contribute to the determination of kinetics. Even at a single point within the contact patch this is true by nature of the Coulomb Friction law. Neglecting elastic effects, if the wheel is supporting lateral loads and turning a corner at the same time, only a single point within the contact patch will be in rolling, the remainder of the patch will be in slip. If the path is instantaneously straight, only a line within the patch will be rolling. Considering elastic rolling bodies, the point and line will expand to small areas of rolling, but still, given loads, these areas will be surrounded by areas of slip. In bulk, the kinematics of rolling and slip at each point within the patch will determine the deviation of actual motion from the ideal rolling constraint. The deviation in the lateral direction is called lateral creep (which can be directly related to sideslip angle) and the projection onto the contact normal of the angular velocity of the wheel relative to its rolling surface is called spin. Longitudinal creep, or deviation from the rolling constraint in the rolling direction, may also exist given non-zero rolling resistance.

For the numerical analysis, we have adopted the algorithm by Shen, Hedrick and Elkins (Shen et.al., 1984) and used it to determine optimal values for the material moduli of elasticity, moduli of rigidity, coefficient of friction, normal force, and geometric parameters. The Shen, Hedrick, and Elkins model applies Kalker's linear (simplified) theory to the tangential contact problem (Johnson, 1987), (Kalker, 1982). This theory assumes that traction force is directly proportional to creep which is an accurate approximation for low values of creep and spin. It also assumes that the normal and tangential contact problems are uncoupled, as the algorithm first computes the normal solution, and then the tangential. Our results are preliminary and pertain only to our current design. However, one resulting design directive that we are now following is to use a circular contact patch, achievable with a sphere-on-sphere geometry. We are adapting hard spheres coated with 
high coefficient of friction materials as wheels by drilling them through and bearing mounting them to axles. The detail in Figure 5 shows an example.

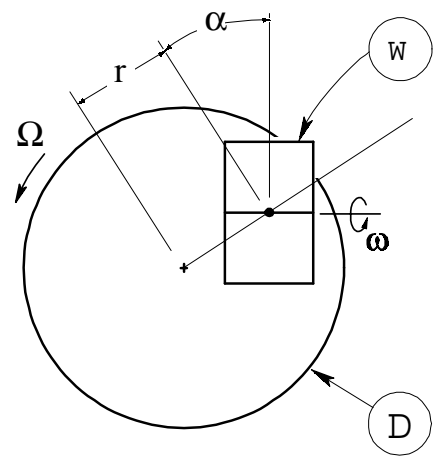

Figure 7: Configuration of Wheel $\mathrm{W}$ rolling on Disk D for numerical and experimental characterization of rolling contact.

Figure 7 shows a wheel $W$ rolling on a Disk $D$. Radius $r$ characterizes the radius of curvature and angle $\alpha$ the sideslip angle of the rolling motion. The full kinematics of rolling, however, require the measurement of the angular velocity of $\omega$ of $W$ and angular velocity $\Omega$ of $D$, which in turn may be related to the location of the areas of stick and slip within the contact patch. When the contact patch is a line, as between a cylindrical wheel $W$ and flat disk $D$ as depicted in Figure 7, there exists a single point in rolling. The relative velocity field for the entire contact line may then be constructed and used with a Coulomb friction model to relate kinetics to kinematics. An attractive manner to express this relationship is to use lateral creep, longitudinal creep, and spin to describe the kinematics. See (Gillespie 2002) for an example of such an analysis.

We have fabricated an instrumented apparatus based on the wheel and disk of Figure 7. Our intentions are to complement the numerical analysis outlined above with an experimental characterization of the rolling contacts between various candidate wheels and rolling surfaces. In the apparatus, disk $D$ is instrumented with an encoder and driven by a motor mounted to ground. Wheel $W$ is loaded by a brake, instrumented with an encoder, and mounted to ground through a six-axis force/torque sensor. Flexible fixturing allows the dimensions $r$ and $\alpha$ and the normal force (contact patch size) to be varied while a semi-automated data acquisition system records normal force, $\mathbf{f}_{\perp}, \mathbf{f}_{\|}$, and $\tau_{\mathrm{s}}$ as functions of longitudinal creep, lateral creep, and spin.

\section{CONCLUSION}

Cobots are intrinsically safe devices that are being developed to cooperate with human users in diverse operations in automobile assembly as well as to assist surgeons in operating rooms. However, they have not yet been designed to assist people with motor impairments. This paper presented the design of an Elbow Cobot that would collaborate with motor impaired persons to achieve limb motions on spherical taskspaces with fixed center. We discussed important features of the Elbow Cobot design, emphasizing the built-in methods for adjusting gimble center-positions and pre-tensioning the normal force on the rolling contact. We briefly discussed our current approach to analysis of the contact patch and optimization of the various design parameters that contribute to ultimate performance. Shortly we will be fabricating our design and conducting initial tests to further adapt it to its intended assistive application.

\section{REFERENCES}

B. Gillespie, J.E. Colgate, and M. Peshkin, "A General Framework for Cobot Control," IEEE Transactions on Robotics and Automation, Vol. 17, No. 4, pp. 391-401, August 2001.

B. Gillespie, C.A. Moore, J.E. Colgate, and M. Peshkin, "Traction Drive Mechanics for Cobots:

Kinematic Creep in Continuously Variable Transmissions," ASME Journal of Mechanical Design, (in press, 2002).

Johnson, K.L., Contact Mechanics (Cambridge University Press, Cambridge, 1987).

Kalker, J.J., Fast Algorithm for the simplified theory of rolling contact, Vehicle System Dynamics Journal, v11, February 1982.

Larsen, HR, "International Journal of Alternative and Complementary Medicine, (1997, Oct), 15(10):22-24.

Peshkin, M, Colgate, JE, "IEEE Robotics \& Automation Magazine, (1996, Dec), 3(4).

M. Peshkin, C. Moore, B. Gillespie and J.E. Colgate, "Cobot Architecture," IEEE Transactions on Robotics and Automation, Vol. 17, No. 4, pp. 377-390, August 2001.

Shen, Z. Y., Hedrick, J.K., and Elkins J.A. (1984), A comparison of alternative creep-force models for rail vehicle dynamical analysis, in L,K. Hedrick, Ed., Proc. $8^{\text {th }}$ IAVSD Symposium on Vehicle System Dynamics, Swets and Zeitlinger, Amsterdam, pp. 591-605.

Stolov, WC, Clowers, MR, "Handbook of Severe Disability”, U.S. Department of Education Rehabilitation Services Administration, (1981), pp. 83101,111-19,137-55. 